\title{
Impact of Mandatory Wake Time on Sleep Timing, Sleep Quality and Rest-Activity Cycle in College and University Students Complaining of a Delayed Sleep Schedule: An Actigraphy Study
}

This article was published in the following Dove Press journal:

Nature and Science of Sleep

\author{
Christophe Moderie ${ }^{1,2}$ \\ Solenne Van der Maren $\mathbb{D}^{1,3}$ \\ Jean Paquet (D) \\ Marie Dumont id ${ }^{1,4}$ \\ 'Center for Advanced Research in Sleep \\ Medicine, Sacre-Coeur Hospital, CIUSSS- \\ NIM, Montreal, Quebec, Canada; \\ ${ }^{2}$ Department of Psychiatry, McGill \\ University, Montreal, Quebec, Canada; \\ ${ }^{3}$ Department of Psychology, University of \\ Montreal, Montreal, Quebec, Canada; \\ ${ }^{4}$ Department of Psychiatry, University of \\ Montreal, Montreal, Quebec, Canada
}

Background: Individuals complaining of a delayed sleep schedule are expected to have shorter sleep duration and lower sleep quality when they must comply with morning obligations. The changes in the sleep schedule imposed by morning obligations may in turn decrease the stability and amplitude of their rest-activity cycle. These expectations were only partially supported in previous studies, possibly due to poor differentiation between days with mandatory or free wake times.

Participants: Fourteen college/university students (8 women) with a complaint of a late sleep schedule and a bedtime after midnight were compared to fourteen controls with an earlier sleep schedule and no complaint.

Methods: During a week of 24-h activity recording, participants specified in their sleep diary whether their wake time was free or determined by an obligation.

Results: The number of nights with mandatory wake times was similar in the two groups. Groups were also similar for sleep duration and sleep quality over the 7 days of recording. Actigraphic sleep efficiency was the same in the two groups for both free and mandatory wake times, but subjective sleep quality decreased on the nights with mandatory wake time in both groups. On the nights with mandatory wake time, delayed participants had shorter sleep episodes and less total sleep time than controls. Rest-activity cycle amplitude was lower in the delayed group whether wake time was free or mandatory.

Conclusion: Sleep duration and total sleep time differed between the two groups only when wake time was mandatory. Prior to mandatory wake times, delayed participants kept the same bedtime and shortened their sleep; sleep latency and sleep efficiency were preserved but subjective sleep quality and alertness on awakening decreased compared to nights with free wake time. Lower amplitude of the rest-activity cycle in delayed subjects may reflect lifestyle differences compared to control participants.

Keywords: sleep schedule, circadian sleep disorders, chronotype, ambulatory recordings, delayed sleep-wake phase disorder, social jetlag

\section{Introduction}

Sleep timing varies greatly from one person to the other. ${ }^{1}$ In a given population, individuals with an evening chronotype (E-types) go to sleep and wake up later than most people. When their delayed sleep schedule impairs their ability to meet social obligations, E-types experience a variety of problems and present a complaint. In severe cases, the complaint associated with delayed sleep may become clinically
Center for Advanced Research in Sleep Medicine (J-5 I85), Sacre-Coeur Hospital of Montreal, CIUSSS-NIM, 5400 Boul.

Gouin Ouest, Montreal, QC H4J IC5, Canada

Tel + I $5143382222, x-2246$

Fax + I 5143382531

Email Marie.dumont@umontreal.ca 
significant and lead to the diagnosis of a circadian sleep disorder called delayed sleep-wake phase disorder (DSWPD). ${ }^{2}$

When they try to follow conventional bedtimes and wake times, both DSWPD patients and E-types complain of a long time to fall asleep and of insufficient sleep duration. ${ }^{2,3}$ Because their sleep episode occurs at an unfavorable circadian phase, sleep efficiency and subjective sleep quality may also decrease compared to individuals with an earlier chronotype. ${ }^{4,5}$ However, observations from field studies are not consistent. Some studies reported shorter sleep duration in delayed individuals compared to controls, ${ }^{3,6-8}$ but others did not find any difference. ${ }^{9-12}$ The same is true for sleep quality measured with actigraphy: some studies found decreased sleep efficiency in delayed individuals, ${ }^{13}$ whereas others reported normal sleep parameters compared to controls. ${ }^{9-12,14}$

Delayed individuals are expected to have normal sleep duration and quality when they can sleep at their spontaneous sleep time. ${ }^{2}$ Therefore, discrepancies in previous studies might be explained by a poor differentiation between nights with or without obligations in the morning. Some studies compared sleep patterns between workdays and weekends in delayed individuals. Using questionnaires, most found shorter sleep duration on weekdays and longer compensatory sleep on weekends. ${ }^{3,6,7,15,16}$ This behavior was also found using actigraphy, ${ }^{8}$ although it was not necessarily specific to delayed individuals. ${ }^{12}$ Beyond sleep timing and duration, few studies have compared sleep quality between work days and days off in delayed individuals. Using a sleep questionnaire, a study found that a later chronotype was associated with lower subjective sleep quality on work days compared to days off. ${ }^{17}$ Using actigraphy, one study on DSWPD patients failed to observe a difference in sleep efficiency or total sleep time between weekdays and weekends, ${ }^{11}$ whereas another study on E-types found decreased sleep efficiency on weekdays. ${ }^{8}$

Comparing weekdays and weekends, however, does not account for morning obligations during the weekend or for obligation-free mornings during the week. Both are common among young adults, especially in college and university students. Many students have some control over their classes schedule, and E-types may avoid classes starting early in the morning. In addition, severity of the sleep delay may lead some DSWPD patients to give up entirely on school or work because they cannot keep up with the imposed starting time in the morning. In fact, in studies on DSWPD patients, many participants were unemployed and their wake times on weekdays were too late for standard starting work or school times. ${ }^{11,14,18}$ Usually, E-types do attempt to follow the schedule imposed by work or school but some of them find it difficult: they often arrive late and they occasionally miss their first class. As a result, these delayed individuals present the main clinical feature of DSWPD which is a complaint that eveningness is a source of problems in their everyday life. ${ }^{9}$ To our knowledge, no previous study directly compared the nights with and without morning obligations in individuals complaining of a delayed sleep schedule.

More eveningness is usually associated with larger differences in sleep timing between work days and days off. ${ }^{17,19,20}$ The concept of "social jetlag" refers to this difference and is quantified by comparing the time of midsleep between free and work days. ${ }^{20}$ Greater social jetlag may increase the overall variability in sleep parameters. Indeed, variability in sleep timing and duration is greater in DSWPD patients than in healthy controls, ${ }^{11}$ and more variability is associated with later circadian phase. ${ }^{21}$ Moreover, increased activity during the night due to more time awake, and decreased activity during the day due to daytime sleepiness may also decrease the amplitude of the rest-activity cycle on work days compared to free days. Increased variability of the rest-activity cycle and blunted amplitude have been associated with adverse health outcomes. $^{22,23}$ To date, the rest-activity profile of individuals complaining of a delayed sleep schedule has been poorly characterized. One study on DSWPD patients ${ }^{14}$ and one on E-types ${ }^{8}$ found activity levels and rest-activity cycle amplitude similar to those of healthy controls or earlier chronotypes, respectively. However, the impact of morning obligations has not been assessed.

In the present study, participants were asked to specify in their sleep diary whether their wake time (WT) that morning was mandatory because of any kind of obligation, or if it was free and spontaneous. This information was used to compare subjective (sleep diaries) and objective (activity recordings) measures of sleep duration and quality between nights with or without obligations in the morning, in individuals complaining of a delayed sleep schedule and in control participants. Stability and amplitude of the 24-h rest-activity cycle were also compared between the two groups. It was expected that the variations in sleep timing and quality between days with or without morning obligations in delayed participants would 
decrease the stability of the 24-h cycle over the week of recording compared to control subjects. For sleep parameters and rest-activity amplitude, the hypothesis was that the negative effects of a delayed sleep schedule as well as differences between delayed participants and controls will be observed specifically when WT was mandatory.

\section{Participants and Methods \\ Participants}

Two groups of 14 college and university students were recruited according to their habitual bedtime and their satisfaction concerning their sleep schedule as reported in a screening interview. Participants in the delayed group (8 women, 6 men, $21.3 \pm 1.2$ years old) had a habitual bedtime after midnight and were complaining of problems caused by their sleep schedule. Habitual bedtime was confirmed using a one-week sleep diary, and the presence of a complaint was confirmed during laboratory screening by a question added at the end of the MorningnessEveningness Questionnaire (MEQ): ${ }^{24}$ "If you consider yourself to be an 'evening' type, does your habitual sleep schedule cause you problems for work or school?". In the presence of problems, volunteers noted those problems as "mild" (8 subjects), "moderate" (5 subjects), or "marked" (1 subject). None evaluated their problems as "severe" or "disabling". The 14 participants included in the control group ( 8 women, 6 men, $22.1 \pm 2.5$ years old) had a habitual bedtime before midnight and no complaint concerning their sleep schedule. All participants were college/ university students, except one control subject who was working full-time at the time of the study. The research project was in accordance with the Declaration of Helsinki and was approved by the Ethics Committee of SacreCoeur Hospital of Montreal. Each participant gave written informed consent. This study is the fourth and last part of a larger research project and more details on subjects' selection can be found in the previous publications. ${ }^{25-27}$ Subjects' characteristics are summarized in Table 1.

\section{Study Protocol}

The research protocol included 7 days of ambulatory recordings followed by a laboratory session that included the measure of salivary dim light melatonin onset (DLMO) to estimate circadian phase. Procedures and results of the laboratory part of the study are presented in a previous publication. $^{25}$
Table I Characteristics of the Participants

\begin{tabular}{|c|c|c|c|c|}
\hline Variable & $\begin{array}{l}\text { Delayed } \\
\text { Subjects }\end{array}$ & $\begin{array}{l}\text { Control } \\
\text { Subjects }\end{array}$ & $p$-value & $d$ \\
\hline $\begin{array}{l}\text { N } \\
\text { Age (y) } \\
\text { Gender (M,F) } \\
\text { MEQ score } \\
\text { PSQI score }\end{array}$ & $\begin{array}{l}14 \\
21.3 \pm 1.2 \\
6,8 \\
38.6 \pm 9.2 \\
4.7 \pm 1.7\end{array}$ & $\begin{array}{l}14 \\
22.1 \pm 2.5 \\
6,8 \\
54.1 \pm 4.8 \\
3.1 \pm 2.3\end{array}$ & $\begin{array}{l}0.30 \\
\mathrm{~N} / \mathrm{A} \\
<0.001 \\
0.053\end{array}$ & $\begin{array}{l}2.11 \\
0.76\end{array}$ \\
\hline $\begin{array}{l}\text { Habitual bedtime } \\
\text { (screening) (h:min } \pm \text { min) } \\
\text { [range] }\end{array}$ & $\begin{array}{l}01: 30 \pm 76 \\
{[00: 19-04: 49]}\end{array}$ & $\begin{array}{l}23: 23 \pm 16 \\
{[22: 51-23: 54]}\end{array}$ & N/A & - \\
\hline $\begin{array}{l}\text { Social jet lag (screening } \\
\text { questionnaire) (h) } \\
\text { [range] }\end{array}$ & $\begin{array}{l}2.4 \pm 1.1 \\
{[1.2-4.1]}\end{array}$ & $\begin{array}{l}1.4 \pm 0.7 \\
{[0.5-2.6]}\end{array}$ & 0.01 & 1.08 \\
\hline $\begin{array}{l}\text { Target bedtime (h:min } \\
\pm \text { min) } \\
\quad \text { [range] }\end{array}$ & $\begin{array}{l}01: 36 \pm 74 \\
{[00: 30-04: 00]}\end{array}$ & $\begin{array}{l}23: 15 \pm 14 \\
{[23: 00-23: 45]}\end{array}$ & N/A & - \\
\hline DLMO (h:min $\pm \min )$ & $23: 03 \pm 87$ & $21: 13 \pm 60$ & 0.001 & 1.47 \\
\hline Interval DLMO-TB (h) & $2.5 \pm 1.0$ & $2.0 \pm 0.9$ & 0.19 & 0.53 \\
\hline
\end{tabular}

Notes: Means $( \pm \mathrm{SD})$ are shown. Group differences assessed using independent samples $t$-tests and effect size (d).

Abbreviations: MEQ, Morningness-Eveningness Questionnaire; PSQI, Pittsburgh Sleep Quality Index; DLMO, dim light melatonin onset; TB, target bedtime; N/A, not applicable (inclusion criteria).

The week of ambulatory recordings was planned to be representative of the participants' habitual sleep schedule. For each participant, a personalized target bedtime (TB) was set for the week of ambulatory recording to avoid deviations due to special occasions. This TB was set using the averaged bedtime reported by the participant during the week of screening sleep diary for which there was no restriction. During the study week of activity recording, participants were asked to go to bed within \pm $60 \mathrm{~min}$ of their TB. Study bedtimes were then compared to screening bedtimes to ensure that they were representative of the habitual sleep schedule of the participants. Mean target bedtime was 01:36 in the delayed group and 23:15 in the control group (Table 1). Wake time was not restricted.

\section{Ambulatory Sleep Assessments Wrist Actigraphy}

Participants wore an actigraph (Actiwatch-2; Philips, Amsterdam, Netherlands) on their non-dominant wrist $24 \mathrm{~h}$ per day, for 7 consecutive days. They removed the actigraph during incompatible activities (showering, swimming, contact sports) and identified those moments in their sleep diary. Actigraphic analyses include 13 subjects per 
group because data could not be retrieved from the monitors of two participants (one delayed and one control).

For the analysis of nighttime sleep, daily bedtimes and wake times were set manually in the data file, after visual inspection of the one-minute data. Decisions were based on abrupt changes in activity and light exposure combined to the clock times indicated by the participants in their sleep diaries and were counterchecked by two investigators (CM and SVM). Other sleep parameters were computed automatically by the dedicated software (Actiware, Philips), using the medium threshold (40 activity counts) to qualify a 1-min epoch as wake. Sleep onset was defined as the beginning of the first 10 consecutive minutes scored "sleep", sleep offset as the beginning of the last 10 consecutive minutes scored "wake", and the duration of the sleep episode was the interval between onset and offset. Other variables computed were: total sleep time (TST), which is the number of minutes scored "sleep" during the sleep episode, sleep efficiency (SE, TST divided by the duration of the sleep episode X 100), and sleep onset latency (SOL, number of minutes elapsed between bedtime and sleep onset).

For the 24-h profiles of activity, daytime data recorded when the monitor was not worn, as reported by the participants or as suggested by 30 consecutive minutes of no activity, were removed from the analyses. Hours with less than 30 minutes of valid data were marked as missing. Data were then averaged over each hour of recording. Nonparametric analysis was performed over the 7 days of recording to estimate interdaily stability (IS) and intradaily variability (IV) ${ }^{28}$ IS estimates how much the pattern of activity is similar from one day to the other. IV estimates the frequency of transitions between activity and rest and reflects the fragmentation of the $24-\mathrm{h}$ rest-activity cycle. To estimate the amplitude of the cycle of each subject, the 1-h means were averaged, first across the 7 days of recording and then separately for the days with mandatory or free WT. The amplitude (AMP) of the resulting profiles was computed as the difference between M10 (the average hourly activity counts for the uninterrupted most active 10 -h period) and L5 (the average hourly activity counts for the uninterrupted least active 5 -h period). ${ }^{29}$

\section{Sleep Diaries}

Participants filled out a short questionnaire every morning and every evening during their week of ambulatory recording. The morning questionnaire was the same as the one used during screening and asked for their bedtime the night before, their wake-up time that morning, and whether their WT was free or mandatory due to any kind of obligation (work, school, planned activity, etc). Two numeric scales also asked to note how well they slept the night before (1: very badly to 5: very well) and how alert and energetic they felt when they got up (1: very tired to 5 : top shape). The evening questionnaire included another numeric scale asking for how alert and energetic they felt in general during that day (1: very tired to 5: top shape). The sleep diary also included a section to write down the moments (if any) when the ambulatory monitor was removed, for how long and why.

\section{Statistical Analysis}

Groups were compared using independent samples $t$-tests. Before analyses, sleep latencies (SOL) were $\log _{10}$ transformed to normalize their distribution. Group-by-night ANOVA assessed the significance of an interaction between the two groups and the nights with free or mandatory WT. Simple effects analyses were conducted when an interaction effect was observed. Group-by-time and night-by-time ANOVA were applied to the 24-h activity profiles to identify time-of-day differences between groups and between nights with free or mandatory wake times, respectively. Simple effects analyses were also conducted when an interaction effect was observed. The correction controlling for the false discovery rate (FDR) was applied to simple effect analyses to adjust significance levels for multiple comparisons. ${ }^{30}$ Effect sizes are also reported for $t$-tests (Cohen's $d$ ) and ANOVA (partial eta-squared, $\eta_{\mathrm{p}}{ }^{2}$ ). According to Cohen, small, medium and large effect sizes are respectively 0.2 , 0.5 and 0.8 for ' $d$ ", and $0.01,0.06$, and 0.14 for $\eta_{\mathrm{p}}{ }^{2} .{ }^{31}$

Analyses were performed using SPSS (version 24, IBM, Armonk, NY, USA). Significance was set at $p \leq 0.05$. Data are presented as mean \pm standard deviation (SD), except when specified otherwise.

\section{Results}

\section{Sleep Timing and Sleep Quality Actigraphic Sleep Variables}

When all days of recording were considered, the two groups of participants differed significantly only for their sleep timing (Table 2). The two groups were similar for TST and SE. Subjective estimates of sleep and alertness quality were also similar in the two groups. 
Table 2 Actigraphic and Subjective Sleep Variables in the Two Groups of Participants

\begin{tabular}{|c|c|c|c|c|}
\hline Sleep Variable & $\begin{array}{l}\text { Delayed Subjects } \\
(n=\mid 3)\end{array}$ & $\begin{array}{l}\text { Control Subjects } \\
(n=\mid 3)\end{array}$ & $p$-value & $d$ \\
\hline Bedtime (h:min $\pm \min$ ) & $01: 36 \pm 64$ & $23: 38 \pm 29$ & $<0.001$ & 2.42 \\
\hline Sleep onset (h:min \pm min) & $0 \mathrm{I}: 48 \pm 65$ & $00: 00 \pm 36$ & $<0.001$ & 2.04 \\
\hline Sleep offset (h:min $\pm \min )$ & $09: 13 \pm 80$ & $07: 59 \pm 57$ & 0.01 & 1.05 \\
\hline Duration of sleep episode $(\mathrm{h})$ & $7.4 \pm 0.8$ & $8.0 \pm 0.8$ & 0.08 & 0.70 \\
\hline Total sleep time $(\mathrm{min})$ & $394 \pm 53$ & $419 \pm 61$ & 0.48 & 0.44 \\
\hline Sleep efficiency (\%) & $88.8 \pm 4.4$ & $87.0 \pm 5.7$ & 0.39 & 0.34 \\
\hline Sleep onset latency $(\min )$ & $11.7 \pm 6.7$ & $21.6 \pm 15.0$ & 0.09 & 0.02 \\
\hline Subjective sleep quality (score) & $4.2 \pm 0.5$ & $4.0 \pm 0.6$ & 0.22 & 0.49 \\
\hline Subjective alertness on awakening (score) & $3.6 \pm 0.6$ & $3.9 \pm 0.6$ & 0.26 & 0.45 \\
\hline Subjective alertness during the day (score) & $3.9 \pm 0.5$ & $4.1 \pm 0.5$ & 0.35 & 0.37 \\
\hline
\end{tabular}

Notes: Means $( \pm S D)$ are shown. Subjective scores from I to 5; higher scores reflect better sleep or alertness quality. Group differences assessed using independent samples $t$-tests and effect size $(d)$.

On average, the number of nights with a mandatory WT was similar in the two groups of participants (delayed: $4.6 \pm 1.6$, controls: $3.9 \pm 1.4 ; \mathrm{p}=0.26$ ). Two participants in each group did not have any night with free WT during the week of ambulatory recording, leaving 11 subjects per group for these analyses. Results of group-by-night ANOVA are presented in Table 3. Bedtime and time of sleep onset were later in delayed subjects, but there was no

Table 3 Actigraphic Sleep Variables for the Nights with Free or Mandatory Wake Times, in the Two Groups of Participants

\begin{tabular}{|c|c|c|c|c|c|}
\hline \multirow[t]{2}{*}{ Sleep Variable } & \multirow{2}{*}{$\begin{array}{l}\text { Delayed Subjects } \\
(n=I I)\end{array}$} & \multirow{2}{*}{$\begin{array}{l}\text { Control Subjects } \\
(n=I I)\end{array}$} & \multicolumn{3}{|c|}{$\begin{array}{l}\text { ANOVA Group x Night } \\
p \text {-value }\left(\eta_{p}{ }^{2}\right)\end{array}$} \\
\hline & & & Group & Night & Interaction $\mathbf{G x N}$ \\
\hline \multicolumn{6}{|c|}{ Bedtime (h:min $\pm \min$ ) } \\
\hline Free WT & $01: 22 \pm 71$ & $23: 45 \pm 25$ & $<0.001$ & 0.50 & 0.23 \\
\hline Mandatory WT & $01: 33 \pm 66$ & $23: 42 \pm 31$ & $(0.53)$ & $(0.02)$ & $(0.07)$ \\
\hline \multicolumn{6}{|c|}{ Sleep onset (h:min $\pm \min$ ) } \\
\hline Free WT & $01: 33 \pm 73$ & $00: 13 \pm 3 \mid$ & $<0.001$ & 0.76 & 0.10 \\
\hline Mandatory WT & $01: 42 \pm 65$ & $00: 00 \pm 38$ & $(0.45)$ & $(0.01)$ & $(0.13)$ \\
\hline \multicolumn{6}{|c|}{ Sleep offset (h:min \pm min) } \\
\hline Free WT & $09: 32 \pm 80$ & $08: 14 \pm 54^{*}$ & 0.05 & 0.12 & 0.05 \\
\hline Mandatory WT & $08: 52 \pm 82$ & $08: 19 \pm 38$ & $(0.19)$ & $(0.12)$ & $(0.18)$ \\
\hline \multicolumn{6}{|c|}{ Duration of sleep episode (h) } \\
\hline Free WT & $8.0 \pm 1.1$ & $8.0 \pm 1.1$ & 0.13 & 0.27 & 0.01 \\
\hline Mandatory WT & $7.2 \pm 1.1$ & $8.4 \pm 0.7 * *$ & $(0.11)$ & $(0.06)$ & $(0.30)$ \\
\hline \multicolumn{6}{|c|}{ Total sleep time $(\mathrm{min})$} \\
\hline Free WT & $426 \pm 62$ & $417 \pm 77$ & 0.36 & 0.25 & 0.01 \\
\hline Mandatory WT & $378 \pm 82$ & $439 \pm 53^{*}$ & $(0.04)$ & $(0.07)$ & $(0.32)$ \\
\hline \multicolumn{6}{|l|}{ Sleep efficiency (\%) } \\
\hline Free WT & $88.8 \pm 3.8$ & $86.4 \pm 6.7$ & 0.54 & 0.76 & 0.39 \\
\hline Mandatory WT & $87.7 \pm 7.8$ & $87.0 \pm 6.2$ & $(0.01)$ & $(0.02)$ & $(0.04)$ \\
\hline \multicolumn{6}{|c|}{ Sleep onset latency $(\mathrm{min})$} \\
\hline Free WT & $11.1 \pm 7.1$ & $28.5 \pm 19.3$ & 0.35 & 0.02 & 0.34 \\
\hline Mandatory WT & $8.9 \pm 3.4$ & $18.3 \pm 15.3$ & $(0.05)$ & $(0.23)$ & $(0.04)$ \\
\hline
\end{tabular}

Notes: Simple effect analysis: ${ }^{*} p \leq 0.05, *^{*} p \leq 0.01$, delayed vs controls.

Abbreviations: WT, wake time; $\eta_{\mathrm{p}}{ }^{2}$, partial eta-squared effect size. 
significant night effect (with free vs mandatory WT) and no group-by-night interaction. Delayed subjects woke up about 40 min later when WT was free compared to mandatory; the group-by-night interaction was significant, showing that sleep offset differed between the two groups only on the nights with free WT. Duration of the sleep episode and TST were shorter in delayed subjects on the nights with mandatory WT, but were quite similar in the two groups on the nights with free WT. SE was the same in the two groups and in the two night conditions. A significant night effect showed that SOL was shorter on the nights preceding mandatory WT in the two groups.

\section{Subjective Sleep Quality}

Subjective sleep quality was lower on the nights with mandatory WT in the two groups (Table 4). Alertness on awakening was significantly worst following mandatory WT compared to free WT, and the interaction effect showed that this difference was larger in delayed participants. Subjective daytime alertness was the same in the two groups and the two night conditions.

\section{4-h Rest-Activity Cycle}

There was no group difference for IS and IV estimates (Table 5). AMP and M10 were significantly lower in delayed than in control subjects but L5 was similar in the two groups. The 24-h profiles for the week of activity recording in the two groups are illustrated in Figure 1. Group-by-time interaction was significant $\left(\mathrm{F}_{23,552}=4.3, \mathrm{p}<0.001\right)$ : activity levels were significantly lower in delayed subjects than in controls at
Table 5 Rest-Activity Cycle Variables in the Two Groups of Participants

\begin{tabular}{|l|l|l|l|l|}
\hline Variables & $\begin{array}{l}\text { Delayed } \\
\text { Subjects } \\
(\mathbf{n}=13)\end{array}$ & $\begin{array}{l}\text { Control } \\
\text { Subjects } \\
(\mathbf{n}=13)\end{array}$ & $p$-value & $d$ \\
\hline IS & $0.48 \pm 0.07$ & $0.51 \pm 0.08$ & 0.36 & 0.36 \\
IV & $0.74 \pm 0.19$ & $0.85 \pm 0.27$ & 0.23 & 0.49 \\
L5 & $15.6 \pm 12.8$ & $16.7 \pm 12.2$ & 0.83 & 0.08 \\
MI0 & $320.7 \pm 78.9$ & $405.6 \pm 84.8$ & 0.01 & 1.04 \\
AMP & $305.1 \pm 73.2$ & $389.0 \pm 80.5$ & 0.01 & 1.09 \\
\hline
\end{tabular}

Notes: Means $( \pm S D)$ are shown. Group differences assessed using independent samples $t$-tests and effect size (d). 13 subjects per group (missing data for one woman in each group).

Abbreviations: IS, interdaily stability; IV, intradaily variability; $L 5$, activity in the low active $5 \mathrm{~h} ; \mathrm{MIO}$, activity in the high active $10 \mathrm{~h}$; AMP, activity amplitude.

18:00, but significantly higher in delayed than in control subjects at 01:00 and 02:00 in the early night.

For AMP, group-by-night ANOVA showed no significant interaction with mandatory or free WT (Table 6). A significant interaction for M10 revealed that lower maximal activity in the delayed group compared to the control group was significant only on the days with free WT. Averaged 24-h activity profiles did not show conditionby-time interaction effects in any of the two groups. Activity profiles for the days with mandatory and free WT in each group are illustrated in Figure 2.

\section{Discussion}

Except for sleep timing, all sleep parameters were similar in delayed and control subjects when morning obligations were not taken into account. Consistent with our

Table 4 Subjective Estimates of Sleep and Alertness Quality for the Nights with Free or Mandatory Wake Times, in the Two Groups of Participants

\begin{tabular}{|c|c|c|c|c|c|}
\hline \multirow[t]{2}{*}{ Subjective Variable } & \multirow{2}{*}{$\begin{array}{l}\text { Delayed Subjects } \\
(n=I I)\end{array}$} & \multirow{2}{*}{$\begin{array}{l}\text { Control Subjects } \\
(n=I I)\end{array}$} & \multicolumn{3}{|c|}{$\begin{array}{l}\text { ANOVA Group x Night } \\
p \text {-value }\left(\eta_{p}{ }^{2}\right)\end{array}$} \\
\hline & & & Group & Night & Interaction $\mathbf{G x N}$ \\
\hline \multicolumn{6}{|l|}{ Sleep quality before } \\
\hline Free WT & $4.5 \pm 0.4$ & $4.0 \pm 0.7$ & 0.47 & 0.03 & 0.50 \\
\hline Mandatory WT & $4.0 \pm 0.7$ & $3.9 \pm 0.8$ & $(0.08)$ & $(0.22)$ & $(0.03)$ \\
\hline \multicolumn{6}{|l|}{ Alertness on awakening } \\
\hline Free WT & $4.2 \pm 0.4^{* * *}$ & $4.0 \pm 0.6$ & 0.46 & $<0.001$ & 0.003 \\
\hline Mandatory WT & $3.1 \pm 0.8$ & $3.8 \pm 0.8$ & $(0.03)$ & $(0.64)$ & $(0.37)$ \\
\hline \multicolumn{6}{|l|}{ Daytime alertness after } \\
\hline Free WT & $4.0 \pm 0.5$ & $4.0 \pm 0.6$ & 0.52 & 0.77 & 0.12 \\
\hline Mandatory WT & $3.7 \pm 0.6$ & $4.1 \pm 0.6$ & $(0.02)$ & $(<0.01)$ & $(0.12)$ \\
\hline
\end{tabular}

Notes: Subjective scores from I to 5; higher scores reflect better sleep or alertness quality. Two participants in each group did not have free WT during their week of sleep diaries. Simple effect analysis: *** $\mathrm{p}<0.00 \mathrm{I}$, free vs mandatory WT.

Abbreviations: WT, wake time; $\eta_{\mathrm{p}}{ }^{2}$, partial eta-squared effect size. 


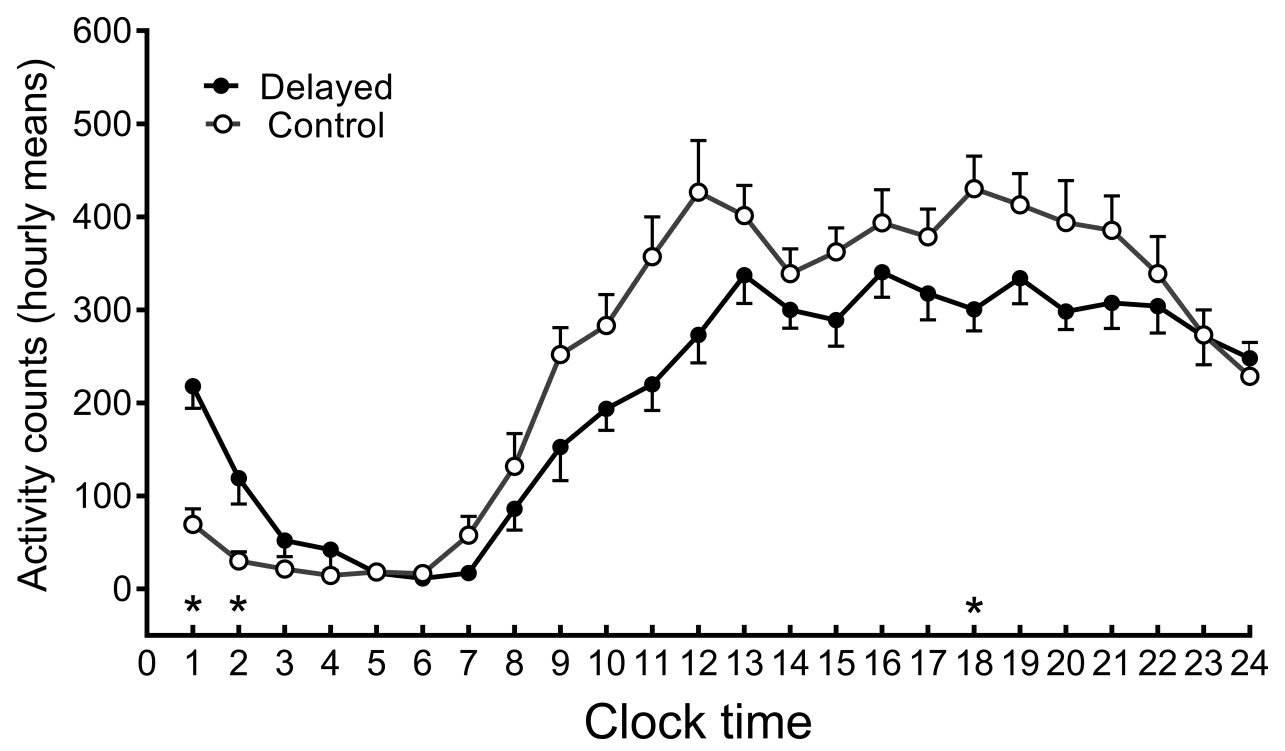

Figure I Rest-activity profiles (hourly means + sem) averaged over the 7 days of activity recording for the delayed (black circles) and control (open circles) subjects. Asterisks indicate significant differences $(p \leq 0.05)$ between the two groups after FDR correction.

hypothesis, the two groups differed only when nights with free or mandatory WT were included in the analyses. On nights with mandatory WT, delayed subjects slept less and for a shorter duration than control subjects. However, SE was almost identical in the two groups and in the two night conditions. SOL did not differ between the two groups and was shorter in both groups on nights with mandatory compared to free WT.

Different strategies might be used by individuals complaining of a delayed sleep schedule when they have commitments in the morning, including 1) go to bed at their spontaneous time and shorten their sleep episode or, 2) go to bed earlier which may increase the time to fall asleep and decrease sleep quality. In the present study, delayed subjects choose the first option. Bedtime was remarkably the same whether they had free or mandatory WT the next morning. By keeping a stable bedtime, delayed participants likely preserved a stable phase relationship between their circadian phase and their sleep episode, preventing internal circadian disruption. This may explain why SOL did not increase and sleep efficiency did not decrease when they had mandatory WT. In fact, SOL was shorter in both groups on the nights with mandatory WT. The clinical significance of this

Table 6 Activity Levels and Estimated Amplitude for the Nights with Free or Mandatory Wake Times, in the Two Groups of Participants

\begin{tabular}{|c|c|c|c|c|c|}
\hline \multirow[t]{2}{*}{ Rest-Activity Cycle Variables } & \multirow{2}{*}{$\begin{array}{l}\text { Delayed Subjects } \\
(n=I I)\end{array}$} & \multirow{2}{*}{$\begin{array}{l}\text { Control Subjects } \\
(n=I I)\end{array}$} & \multicolumn{3}{|c|}{$\begin{array}{l}\text { ANOVA Group x Night } \\
\text { p-value }\left(\eta_{p}{ }^{2}\right)\end{array}$} \\
\hline & & & Group & Night & Interaction $\mathbf{G x N}$ \\
\hline \multicolumn{6}{|l|}{ L5 } \\
\hline Free WT & $15.8 \pm 17.4$ & $15.8 \pm 13.5$ & 0.58 & 0.16 & 0.58 \\
\hline Mandatory WT & $27.9 \pm 29.5$ & $21.2 \pm 14.8$ & $(0.02)$ & $(0.10)$ & $(0.02)$ \\
\hline \multicolumn{6}{|l|}{ MIO } \\
\hline Free WT & $287 \pm 68$ & $428 \pm 85^{* * *}$ & 0.006 & 0.58 & 0.04 \\
\hline Mandatory WT & $329 \pm 127$ & $403 \pm 69$ & $(0.32)$ & $(0.02)$ & $(0.19)$ \\
\hline \multicolumn{6}{|l|}{ AMP } \\
\hline Free WT & $271 \pm 61$ & $413 \pm 82$ & 0.004 & 0.99 & 0.10 \\
\hline Mandatory WT & $301 \pm 133$ & $382 \pm 64$ & $(0.35)$ & $(<0.001)$ & $(0.13)$ \\
\hline
\end{tabular}

Notes: Two participants in each group did not have free WT during their week of sleep diaries. Simple effect analysis: $* * * p=0.00 \mathrm{I}$, delayed vs controls.

Abbreviations: WT, wake time; L5, activity in the low active $5 \mathrm{~h} ; \mathrm{MI0}$, activity in the high active 10 h; AMP, activity amplitude; $\eta_{\mathrm{p}}{ }^{2}$, partial eta-squared effect size. 


\section{Delayed subjects}

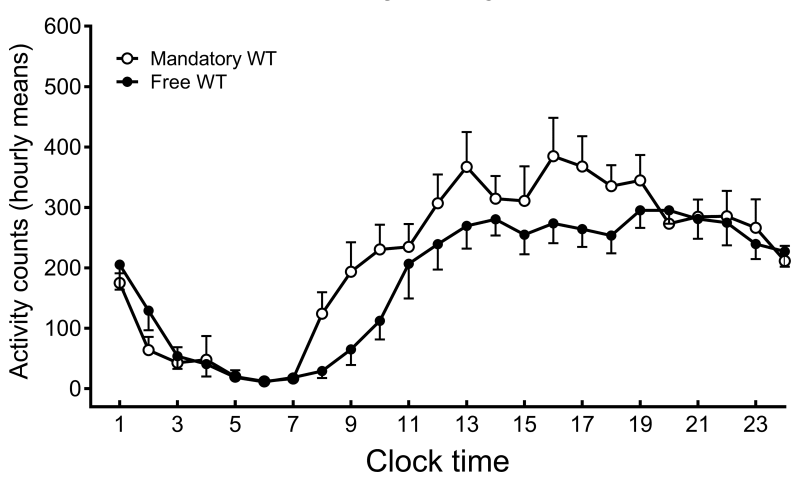

Control subjects

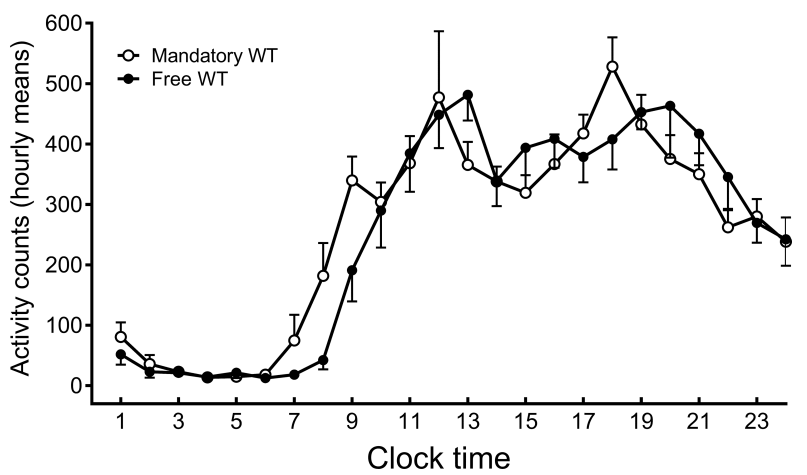

Figure 2 Illustration of the rest-activity cycle (hourly means + sem) for days with mandatory (open circles) or free (black circles) wake times, in the groups of delayed (left panel) and control (right panel) participants.

observation is doubtful, however, since the differences in the number of minutes were small, and group means were shorter than the proposed 30-min criteria for sleep-onset insomnia. $^{32}$

The downside of the option chosen by delayed participants was a reduction of TST and sleep duration on the nights with mandatory WT. However, when compared to controls, there was no apparent compensatory sleep on nights with free WT. In fact, on the nights with free WT, mean sleep duration was exactly the same in the two groups. If sleep restriction on work days in delayed individuals was often observed in previous studies, the presence of compensatory sleep on free days is unclear. Questionnaire studies have reported that individuals with a late chronotype compensate for the sleep debt accumulated during work days by lengthening their sleep on free days by several hours. ${ }^{3,16}$ However, an actigraphy study in college students found shorter sleep duration on weekdays compared to weekends in E-types but no difference in sleep duration between E-types and earlier chronotypes on weekends. ${ }^{8}$ The absence of compensatory sleep on free days could be related to the nature of obligations on work days that limits sleep restriction in student populations. In the present study, average time of sleep offset in delayed participants on days with mandatory WT was $08: 52$, which is relatively late for standard social obligations. This suggests that delayed participants may have adapted their school start time to accommodate their needs. As a result, sleep restriction on days with mandatory WT may have been too small to require significant oversleep on free days. It could be expected that when those individuals reach the work market, schedule accommodation will become more difficult and sleep restriction on work days may become more severe, ${ }^{33}$ increasing the need for compensatory sleep on free days. Questionnaires studies that included larger and more diverse populations probably captured the reality of individuals who have to comply with strict working start times.

Subjective alertness on awakening decreased significantly on the days with mandatory compared to free WT in delayed subjects. This was expected, due to curtailed sleep on these days and because sleep offset occurred at a circadian time of higher sleep propensity. Sleep inertia may also contribute to explaining reduced alertness in delayed participants after mandatory WT. ${ }^{34}$ Sleep restriction and higher circadian sleep propensity amplify the effects of sleep inertia on vigilance levels, ${ }^{35}$ and both late chronotypes and DSWPD patients may present greater sleep inertia after forced awakenings compared to individuals with an earlier sleep schedule. ${ }^{16,36}$

Subjective sleep quality was lower for the nights with mandatory WT in the two groups, which is interesting considering that sleep efficiency measured with actigraphy was identical in the two night conditions. Differences between objective and subjective evaluation of sleep quality have also been observed in previous studies on E-types and DSWPD patients. ${ }^{10,12}$ As suggested by others, ${ }^{12}$ the degree of morning sleepiness could influence the perception of sleep quality when the subjective evaluation is made shortly after waking up. It should be noted from Table 4 that, when compared to the control group, the difference between nights with free or mandatory WT in delayed participants seems mostly due to a very good subjective sleep quality when WT was free rather than a low subjective sleep quality when WT was mandatory. This may reflect the better refreshing quality of sleep felt by delayed subjects when they are allowed to sleep as long as they want. This improved subjective sleep quality was 
not captured by the actigraphic measure of sleep efficiency. It is well-known from previous studies that actigraphic measures do not correlate well with subjective sleep quality. Differences between subjective and objective measures may relate to the limitations of actigraphy, ${ }^{37}$ but they may also reflect the fact that the two measures capture different aspects of the sleep experience. ${ }^{38,39}$

Contrary to our hypothesis, the group difference in social jetlag as reported in the screening questionnaire (Table 1) did not introduce more variability in the rest-activity cycle of delayed participants compared to controls, as shown by similar IS in the two groups. The screening questionnaire and actigraphy probably assessed two different aspects of the participants' sleep behavior. The screening questionnaire asked for a range of bedtimes and wake times for work/ school days and for free/vacation days. The range reported by the volunteers therefore included their vacation sleep times, which often occur at a later time than when the subjects have only one or two free days. Furthermore, during the week of actigraphy, the focus was on determinants of WT and not on free vs work days. Especially in students' populations, a school day may have a free WT if classes are only in the afternoon. Conversely, a free day may have a mandatory WT because of the scheduling of some leisure activity. During the week of activity recording, variations in the time of sleep offset may not have been large enough in the delayed group to introduce detectable variability, and the small number of days with free WT limited the strength of the analyses of variability. A longer duration of ambulatory recordings would be more appropriate to explore the variability of the rest-activity cycle in delayed individuals.

Lower amplitude of the rest-activity cycle of delayed compared to control participants appears to be the consequence of lower maximal daytime activity levels (M10) in the delayed group. Decreased daytime activity may reflect the presence of some daytime napping to compensate for the sleep restriction on the days with mandatory WT. Lower daytime activity could also be a consequence of different lifestyles in the two groups. For example, previous studies have reported an association between greater eveningness and decreased physical activity. ${ }^{7,40}$ Also, increased daily screen time and other poor lifestyle behaviours have been observed in adolescents with later chronotypes. ${ }^{41}$ We already reported that delayed participants in our research project made greater use of computers than controls in the 3 $\mathrm{h}$ before bedtime. ${ }^{26}$ However, whether this behaviour reflects a general preference for sedentary activities in delayed individuals is unknown. More studies are needed to address the causes and consequences of decreased amplitude of the restactivity cycle in delayed individuals.

The main strength of this study is to have specific identification of mornings with free or mandatory WT. Mandatory WT were not limited to weekdays and could also occur on weekends, as highlighted by the observation that two participants in each group did not have any free WT during the week of ambulatory recording. Conversely, free WT can occur on weekdays, especially in student populations. Nevertheless, it would have been of interest to have more information concerning the nature and the timing of morning obligations in the delayed group. An obligation at 10:00 in the morning could mean a mandatory WT for a delayed person but not for an individual with an earlier chronotype. Another strength of the study was that sleep parameters were evaluated both subjectively in sleep diaries, and objectively with actigraphy. These two modes of evaluation provided complementary information and may reflect different aspects of the same reality, especially for sleep quality.

A limitation of the study was that bedtime was not entirely free during ambulatory recordings because of the imposed target bedtime (TB). However, means and SD for bedtime were quite similar during the screening week (Table 1) and the study week (Table 2), both for delayed (screening: 01:30 $\pm 76 \mathrm{~min}$; study: 01:36 $\pm 64 \mathrm{~min}$ ) and control (screening: $23: 23 \pm 16 \mathrm{~min}$; study: $23: 38 \pm 29 \mathrm{~min}$ ) participants. With the range allowed for bedtime (TB \pm 1h), participants had the possibility to go to sleep up to one hour earlier or one hour later than their TB, but on average the bedtime difference between nights with free or mandatory WT was less than $10 \mathrm{~min}$. We are therefore confident that the results were not affected by TB and that the bedtimes observed during the week of ambulatory recordings represent accurately the usual behavior of the participants. By excluding special occasions and vacation times, the sleep schedule of delayed participants showed more stability than expected, considering the large social jetlag reported in the screening questionnaire. Therefore, the results from the week of activity recording may apply only to typical weeks. Longer activity recordings including work/school, free and vacation days would be necessary to be really representative of the general sleep behavior of delayed individuals. Another limitation of the study is the relatively small contrast in sleep timing between the two groups of participants compared to studies on DSPWD patients. However, the advantage of studying this subclinical population of delayed individuals is that all participants were engaged in social obligations (work or 
school). In previous studies where most participants were unemployed, ${ }^{11,14,18}$ comparisons between weekdays and weekends were less informative concerning the impact of mandatory WT in delayed individuals.

\section{Conclusion}

In conclusion, proper identification of mandatory WT in delayed individuals brought out effects of social obligations on sleep and alertness that were obscured when all days were considered together. When facing mandatory WT in the morning, delayed participants choose to keep their bedtime constant and to shorten their sleep instead of trying to fall asleep earlier. This strategy led to some sleep restriction but preserved sleep efficiency and prevented internal circadian disruption. The low amplitude of the rest-activity cycle observed in delayed participants, independent of morning obligations, will need replication in further studies. Low circadian amplitude has been associated with adverse health conditions. ${ }^{42-44}$ Therefore, if confirmed, causes and consequences of low amplitude of the rest-activity cycle in delayed individuals will need to be addressed.

\section{Acknowledgments}

This study was supported by a Research Grant (\#155406-211) from the Natural Sciences and Engineering Research Council of Canada (NSERC) and by student fellowships from the Faculté de Médecine (Université de Montréal), Fonds de la recherche du Québec-Santé (FRQ-S), and NSERC.

\section{Disclosure}

Ms Solenne Van der Maren reports Student Fellowship from Natural Sciences and Engineering Research Council of Canada (NSERC), during the conduct of the study. Professor Marie Dumont reports grants from Natural Sciences and Engineering Research Council of Canada (NSERC), during the conduct of the study. The authors report no other conflicts of interest in this work.

\section{References}

1. Roenneberg T, Kuehnle T, Juda M, et al. Epidemiology of the human circadian clock. Sleep Med Rev. 2007;11:429-438. doi:10.1016/j. smrv.2007.07.005

2. American Academy of Sleep Medicine. The International Classification of Sleep Disorders, Diagnostic and Coding Manual. 3rd ed. Westchester, IL: American Academy of Sleep Medicine; 2014.

3. Roepke SE, Duffy JF. Differential impact of chronotype on weekday and weekend sleep timing and duration. Nat Sci Sleep. 2010;2:213-220.
4. Dijk DJ, Czeisler CA. Contribution of the circadian pacemaker and the sleep homeostat to sleep propensity, sleep structure, electroencephalographic slow waves, and sleep spindle activity in humans. $J$ Neurosci. 1995;15(5):3526-3538. doi:10.1523/JNEUROSCI.15-05-03526.1995

5. Richardson GS. The human circadian system in normal and disordered sleep. J Clin Psychiatry. 2005;66 Suppl 9(suppl 9):3-9.

6. Soehner AM, Kennedy KS, Monk TH. Circadian preference and sleep-wake regularity: association with self-report sleep parameters in daytime-working adults. Chronobiol Int. 2011;28(9):802-809. doi:10.3109/07420528.2011.613137

7. Haraszi RA, Purebl G, Salavecz G, et al. Morningness-eveningness interferes with perceived health, physical activity, diet and stress levels in working women: a cross-sectional study. Chronobiol Int. 2014;31(7):829-837. doi:10.3109/07420528.2014.911188

8. Vitale JA, Roveda E, Montaruli A, et al. Chronotypes influences activity circadian rhythm and sleep: differences in sleep quality between weekdays and weekend. Chronobiol Int. 2015;32 (3):405-415. doi:10.3109/07420528.2014.986273

9. Chang A-M, Reid KJ, Gourineri R, Zee PC. Sleep timing and circadian phase in delayed sleep phase syndrome. J Biol Rhythms. 2009;24 (4):313-321. doi:10.1177/0748730409339611

10. Martin JS, Hébert M, Ledoux É, Gaudreault M, Laberge L. Relationship of chronotype to sleep, light exposure, and work-related fatigue in student workers. Chronobiol Int. 2012;29(3):295-304. doi:10.3109/ 07420528.2011 .653656

11. Burgess HJ, Park M, Wyatt JK, Rizvydeen M, Fogg LF. Sleep and circadian variability in people with delayed sleep-wake phase disorder versus healthy controls. Sleep Med. 2017;34:33-39. doi:10.1016/j. sleep.2017.02.019

12. Saxvig IW, Wilhelmsen-Langeland A, Pallesen S, Nordhus IH, Vedaa O, Bjorvatn B. Habitual sleep, social jetlag, and reaction time in youths with delayed sleep-wake phase disorder. A case-control study. Front Psychol. 2019;10:2569. doi:10.3389/fpsyg.2019.02569

13. Lehnkering H, Siegmund R. Influence of chronotype, season, and sex of subject on sleep behavior of young adults. Chronobiol Int. 2007;24 (5):875-888. doi:10.1080/07420520701648259

14. Joo EY, Abbott SM, Reid KJ, et al. Timing of light exposure and activity in adults with delayed sleep-wake phase disorder. Sleep Med. 2017;32:259-265. doi:10.1016/j.sleep.2016.09.009

15. Taillard J, Philip P, Bioulac B. Morningness/eveningness and the need for sleep. J Sleep Res. 1999;8(4):291-295. doi:10.1046/j.13652869.1999.00176.x

16. Roenneberg T, Wirz-Justice A, Merrow M. Life between clocks: daily temporal patterns of human chronotypes. J Biol Rhythms. 2003;18(1):80-90. doi:10.1177/0748730402239679

17. Pilz LK, Keller LK, Lenssen D, Roenneberg T. Time to rethink sleep quality: PSQI scores reflect sleep quality on workdays. Sleep. 2018;41(5):zsy029. doi:10.1093/sleep/zsy029

18. Solheim B, Langsrud K, Kallestad H, Engstrom M, Bjorvatn B, Sand T. Sleep structure and awakening threshold in delayed sleep-wake phase disorder patients compared to healthy controls. Sleep Med. 2018;46:61-68. doi:10.1016/j.sleep.2018.03.001

19. Juda M, Vetter C, Roenneberg T. Chronotype modulates sleep duration, sleep quality, and social jet lag in shift-workers. J Biol Rhythms. 2013;28(2):141-151. doi:10.1177/0748730412475042

20. Wittmann M, Dinich J, Merrow M, Roenneberg T. Social jetlag: misalignment of biological and social time. Chronobiol Int. 2006;23 (1-2):497-509. doi:10.1080/07420520500545979

21. Murray JM, Phillips AJK, Magee M, et al. Sleep regularity is associated with sleep-wake and circadian timing, and mediate daytime function in delayed sleep-wake phase disorder. Sleep Med. 2019;58:93-101. doi:10.1016/j.sleep.2019.03.009

22. Luik AI, Zuurbier LA, Hofman A, Van Someren EJW, Tiermeier H. Stability and fragmentation of the activity rhythm across the sleep-wake cycle: the importance of age, lifestyle, and mental health. Chronobiol Int. 2013;30(10):1223-1230. doi:10.3109/07420528.2013.813528 
23. Rutters F, Lemmens SG, Adam TC, et al. Is social jetlag associated with an adverse endocrine, behavioral, and cardiovascular risk profile? J Biol Rhythms. 2014;29(5):377-383. doi:10.1177/0748730 414550199

24. Horne JA, Ostberg O. A self-assessment questionnaire to determine morningness-eveningness in human circadian rhythms. Int J Chronobiol. 1976;4(2):97-110.

25. Moderie C, Van der Maren S, Dumont M. Circadian phase, dynamics of subjective sleepiness and sensitivity to blue light in young adults complaining of a delayed sleep schedule. Sleep Med. 2017;34:148-155. doi:10.1016/j.sleep.2017.03.021

26. Van der Maren S, Moderie C, Duclos C, Paquet J, Daneault V, Dumont M. Daily profiles of light exposure and evening use of light-emitting devices in young adults complaining of a delayed sleep schedule. J Biol Rhythms. 2018;33(2):192-202. doi:10.1177/ 0748730418757007

27. Moderie C, Van der Maren S, Paquet J, Dumont M. Home versus laboratory assessments of melatonin production and melatonin onset in young adults complaining of a delayed sleep schedule. J Sleep Res 2019;30:e12905.

28. Van Someren EJW, Swaab DF, Colenda CC, Cohen W, McCall WV, Rosenquist PB. Bright light therapy: improved sensitivity to its effects on rest-activity rhythms in alzheimer patients by application of nonparametric methods. Chronobiol Int. 1999;16(4):505-518. doi:10.3109/07420529908998724

29. Huang Y-L, Liu RY, Wang QS, Van Someren EJW, Xu H, Zhou J-N. Age-associated difference in circadian sleep-wake and rest-activity rhythms. Physiol Behav. 2002;76(4-5):597-603. doi:10.1016/S00319384(02)00733-3

30. Benjamini Y, Hochberg Y. Controlling the false discovery rate: a practical and powerful approach to multiple testing. $R$ Stat Soc B. 1995;57:289-300.

31. Cohen J. Statistical Power Analysis for the Behavioral Sciences. 2nd ed. Hillsdale, NJ: Lawrence Erlbaum Associates; 1988.

32. Lichstein KL, Durrence HH, Taylor DJ, Bush AJ, Riedel BW. Quantitative criteria for insomnia. Behav Res Ther. 2003;41 (4):427-445. doi:10.1016/S0005-7967(02)00023-2

33. Asaoka S, Komada Y, Aritake S, Morita Y, Fukuda K, Inoue Y. Effect of delayed sleep phase during university life on the daytime functioning in work life after graduation. Sleep Med. 2014;15(9):1155-1158. doi:10.1016/j.sleep.2014.05.017
34. Trotti LM. Waking up is the hardest thing i do all day: sleep inertia and sleep drunkenness. Sleep Med Rev. 2017;35:76-84. doi:10.1016/ j.smrv.2016.08.005

35. Tassi P, Muzet A. Sleep inertia. Sleep Med Rev. 2000;4(4):341-353. doi:10.1053/smrv.2000.0098

36. Solheim B, Olsen A, Kallestad H, et al. Cognitive performance in DSWPD patients upon awakening from habitual sleep compared with forced conventional sleep. J Sleep Res. 2019;28(5):e12730. doi:10.1111/jsr. 12730

37. Marino M, Rueschman MN, Winkelman JW, et al. Measuring sleep: accuracy, sensitivity, and specificity of wrist actigraphy compared to polysomnography. Sleep. 2013;36(11):1747-1755. doi:10.5665/ sleep. 3142

38. Aili K, Astrom-Paulsson S, Stoetzer U, Svartengren M, Hillert L. Reliability of actigraphy and subjective sleep measurements in adults: the design of sleep assessments. J Clin Sleep Med. 2017;13 (01):39-47. doi:10.5664/jcsm.6384

39. Faerman A, Kaplan KA, Zeitzer JM. Subjective sleep quality is poorly associated with actigraphy and heart rate measures in community-dwelling older men. Sleep Med. 2020. doi:10.1016/j. sleep.2020.04.012

40. Hisler GC, Phillips AL, Krizan Z. Individual differences in diurnal preference and time-of-exercise interact to predict exercise frequency. Ann Behav Med. 2017;51(3):391-401. doi:10.1007/s12160-016-9862-0

41. Gariépy G, Doré I, Whitehead RD, Elgar FJ. More than just sleeping in: a late timing of sleep is associated with health problems and unhealthy behaviours in adolescents. Sleep Med. 2019;56:66-72. doi:10.1016/j.sleep.2018.10.029

42. Van Someren EJW, Mirmiran M, Swaab DF. Non-pharmacological treatment of sleep and wake disturbances in aging and alzheimer's disease: chronobiological perspectives. Behav Brain Res. 1993;57 (2):235-253. doi:10.1016/0166-4328(93)90140-L

43. Pati AK, Parganiha A, Kar A, Soni R, Roy S, Choudhary V. Alterations of the characteristics of the circadian rest-activity rhythm of cancer in-patients. Chronobiol Int. 2007;24(6):1179-1197. doi:10.1080/07420520701800868

44. Chan-Thim E, Dumont M, Moullec G, et al. Amplitude of the rest-activity cycle in chronic obstructive pulmonary disease: an exploratory study. ChronoPhysiol Ther. 2016;6:75-83. doi:10.2147/ CPT.S117684

\section{Publish your work in this journal}

Nature and Science of Sleep is an international, peer-reviewed, open access journal covering all aspects of sleep science and sleep medicine, including the neurophysiology and functions of sleep, the genetics of sleep, sleep and society, biological rhythms, dreaming, sleep disorders and therapy, and strategies to optimize healthy sleep.
The manuscript management system is completely online and includes a very quick and fair peer-review system, which is all easy to use. Visit http://www.dovepress.com/testimonials.php to read real quotes from published authors. 